\title{
Fluoroquinolones Resistance Salmonella: State of Knowledge
}

\author{
Nazek Al Gallas* and Ridha Ben Aissa \\ Assistant Professor \& Researcher, Food \& Water Control Lab, Pasteur Institute of Tunis, Tunisia
}

Submission: October 24, 2017; Published: December 06, 2017

*Corresponding author: Nazek Al Gallas, Assistant Professor \& Researcher, Water \& Food Control Lab- National Center of Salmonella, Shigella, Vibrio-Enteropathogens, Pasteur Institute of Tunis, 13, Place Pasteur-B.P. 74, 1002 Tunis-Belvédère, Tunisia, Tel: (+216) 71 841 203;

Email: nazek_gallas@yahoo.fr/nazek.algallas@pasteur.rns.tn

\section{Introduction}

Non typhoidal Salmonella (NTS) are one of the principal pathogens implicated in food-borne gastroenteritis worldwide. Antimicrobial agents are not usually essential for the treatment of most cases of salmonellas is but can be lifesaving in cases of severe or systemic infection. According to the World Health Organization, there are about 17 million cases annually of acute gastroenteritis or diarrhea due to non typhoidal salmonellas is, with 3 million deaths. NTS is also among the most common causes of invasive bacterial childhood disease in which antimicrobial chemotherapy can be lifesaving. Antimicrobial resistance to several classes of traditional first-line drugs has emerged in recent decades [1-4].

Owing to the absence of an ecological barrier of human and animal ecosystems as well as the zoonotic property of Salmonella the transit of quinolone-resistant Salmonella isolates to humans is a major health problem. This problem could even be more significant among humans with critical immunological status and would require antibacterial therapeutic intervention [1].

Fluoroquinolones are broad-spectrum antimicrobial drugs used to treat many clinical infections. Salmonellosis is treated with fluoroquinolones (FQs) only in elderly or immune compromised patients, but these drugs are also used for treating patients with enteric fever, invasive disease, or longterm salmonella carriage. Unfortunately, NTS with reduced fluoroquinolones susceptibility has increased during recent years in many countries. The emergence of quinolone-resistant non-typhoidal Salmonella varies by serotype and geographic location. Therefore, the control of quinolone-resistant NTS infection is difficult [1-4].

There is a high need to understand the quinolone resistance mechanisms for preventing the further quinolone-resistance Salmonella development. Thus, prevent the spread of quinoloneresistant Salmonella between humans and animal reservoirs along the food chain $[1,4]$.
What Should We Know About Salmonella Quinolone Resistance Mechanisms

As in other Gram-negative bacteria, mechanisms of resistance to quinolones in Salmonella include target gene mutations, active efflux, and decreased outer membrane permeability. However, the exact contribution of these individual mechanisms to resistance, which may nevertheless interplay to reach highlevel resistance, has not yet clearly been defined as in other bacteria such as Escherichia coli.

\section{Target-Site Mutation}

FQs are direct inhibitors of bacterial DNA synthesis. This is done by inhibiting 2 enzymes: DNA gyrase (Gramnegative bacteria), and topoisomerase IV (Gram-positive microorganisms). In Salmonella spp., these mutations are related to resistance to nalidixic acid (NAL) and reduced susceptibility to FQs such as that of ciprofloxacin (Cip). The main mechanism of $\mathrm{FQ}$ resistance is due to mutation in target genes (gyrA, gyrB, $\operatorname{par} C$, and parE) that encode the primary and secondary target enzymes of these drugs $[1,4,5]$.

In Salmonella, quinolone resistance was firstly attributed to point mutations in the gyrA gene coding for the A subunit of gyrase, whose complex with DNA is the primary target of quinolones. Resistance mutations of gyrA have been clustered in a region of the gene product between amino acids 67 and 106, termed the quinolone resistance-determining region (QRDR). Amino acid changes at Ser-83 (to Phe, Tyr, or Ala) or at Asp-87 (to Gly, Asn, or Tyr) are the most frequently observed in nalidixic acid-resistant strains. Whereas mutations in both S83 and A87 in GyrA are linked with ciprofloxacin-resistant Salmonella isolates from humans and animals. Resistance linked to GyrB mutations (S464T) has been reported only in a single clinical isolate. Mutations in S80 or G78 of ParC have been found in ciprofloxacin-resistant clinical and laboratory-induced isolates. 
Introducing into the respective mutants the corresponding plasmid-encoded quinolone-susceptible wild-type alleles of Escherichia coli gyrA, gyrB, or parC resulted in the reduction of quinolone resistance, indicating a role for these mutations in quinolone resistance. No mutations in parE have yet been described in quinolone-resistant Salmonella. Less frequently, amino acid substitutions at other GyrA positions than Ser-83 and Asp-87 have been described in quinoloneresistant Salmonella strains such as those at Ala-67 (to Pro), Gly-81 (to Ser), and Ala119 (to Glu). Unlike in E. coli, no mutations have been described in the secondary target for quinolones, namely the parC gene coding for the ParC subunit of topoisomerase IV. It remains to be investigated what the specific role of these mutations on quinolone resistance in Salmonella [1,5].

\section{Active Efflux}

Chromosomal multidrug efflux pumps are capable of actively removing FQs and a broad range of antimicrobial agents from the bacterial cell and are mostly encoded by chromosomal genes. At least six major groups of active drug efflux pump transporters have been identified in prokaryotes to date: ATP-binding cassette (ABC), major facilitator super family (MFS), small multidrug resistance (SMR), multi-antimicrobial resistance (MAR), resistance nodulation division (RND), and multidrug and toxic compound extrusion (MATE) [1,5].

Although, in Salmonella, evidence for the participation of active efflux in quinolone resistance has recently been provided; but it had been described in many clinically important bacteria including Campylobacter jejuni (CmeABC), Escherichia coli (AcrAB-TolC, AcrEF-TolC, EmrB, and EmrD), Pseudomonas aeruginosa (MexAB-OprM, MexCD-OprJ, MexEFOprN, and MexXY-OprM), Streptococcus pneumoniae (PmrA), Staphylococcus aureus (NorA) and others $[1,5]$.

The AcrAB-TolC system in Salmonella consists of the cytoplasmic membrane localized AcrB and the accessory protein AcrA, linking AcrB with the outer membrane protein (OMP) TolC. The over expression of AcrAB-tolC is mediated by the transcriptional activators MarA and SoxS, which are regulated by MarR and SoxR proteins. Nevertheless, there is no direct evidence to demonstrate the role of the AcrAB-TolC efflux system in quinolone-resistant Salmonella; therefore, substantial work remains to be done to understand the role of efflux and its regulation in Salmonella [1,3].

\section{Decreased Outer Membrane Permeability}

Very few researches have investigated on alterations of outermembrane proteins expression or the role of lipopolysaccharide composition in quinolone-resistant Salmonella isolates. The lengthening of the $\mathrm{O}$ chains has been studied in quinoloneresistant Salmonella that could also lead to a lower level in the permeability of the outer membrane. Lack of expression of the OmpF porin has been reported in some quinoloneresistant Salmonella strains. However, it was not clear whether the lack of OmpF contributed to decreased levels of quinolone accumulation. It has been shown for Salmonella that SoxS up regulates micF transcription which may be responsible for the lack of expression of $\mathrm{OmpF}$ for these quinolone resistant Salmonella isolates $[1,3,4]$.

\section{Transmissible Quinolone-Resistance Mechanisms}

Plasmid-mediated quinolone resistance (PMQR) genes on mobile genetic elements are able to reduce susceptibility of quinolone or FQ antimicrobials. The PMQR gene, $q n r$, encodes a pentapeptide repeat motif protein $(Q n r)$ that protects the target enzyme DNA gyrase and topoisomerase IV by blocking the quinolone inhibition. Recently, several Qnr proteins were investigated in Enterobacteriaceae (QnrA, QnrB, QnrC, QnrD, $Q n r S)$. The prevalence of qnrSgenes is higher than the other $q n r$ genes in Salmonella. The qnrD gene also has been investigated in eight different Salmonella serovars from 13 European countries $[3,4]$.

Another plasmid-encoded quinolone resistance determinant is a variant of an aminoglycoside acetyl transferase gene, a ac ( $\left.6^{\prime}\right)$ $I b$-cr.The $\mathrm{cr}$ variant of $a a c\left(6^{\prime}\right)-I b$ encodes an aminoglycoside acetyltransferase that confers reduced susceptibility to ciprofloxacin by $\mathrm{N}$-acetylation of its piperazinyl amine. Aac (6')Ib-cr has two amino acid changes, Trp102Arg and Asp179Tyr, which together are necessary and sufficient for the enzyme's ability to acetylate ciprofloxacin. When both $q n r A$ and $a a c\left(6^{\prime}\right)-I b-$ $c r$ are present in the same cell, the level of resistance is increased fourfold more than that conferred by qnrA alone, with an MIC of ciprofloxacin of $1.0 \mu \mathrm{g} / \mathrm{ml}$, a value near the clinical breakpoint for susceptibility. In addition, the presence of aac(6')-Ib-cr alone increased substantially the frequency of selection of chromosomal mutants upon exposure to ciprofloxacin [2-4].

The problem is very urgent, and risks of the overuse and misuse of FQs in food-animal production can contribute to higher levels of resistance in human Salmonella infections. The diversity of quinolone-specific resistance mechanisms that emerged following ciprofloxacin introduction points to an urgent need for therapeutic alternatives and resistance containment. Ensuring early but controlled access to novel antimicrobials may be necessary to ensure medium-to long-term efficacy in resourcelimited settings. Monitoring, preventing, controlling and collaboration among different sectors is strongly recommended.

\section{References}

1. Kongsoi S, Nakajima C, Suzuki Y (2017) Quinolone Resistance in Nontyphoidal Salmonella. In: Mihai Mares (Ed.), Salmonella, Salmonellosis. InTech.

2. Yuan J, Guo W (2017) Mechanisms of resistance to quinolones in Salmonella Typhimurium from patients with infectious diarrhea. Microbiol Immunol 61(3-4): 138-143.

3. Wasyl D, Hoszowski A, Zajac M (2014) Prevalence and characterisation of quinolone resistance mechanisms in Salmonellaspp. Vet Microbiol 171(3-4): 307-314. 
4. Al Gallas N, Abbassi MS, Gharbi B, Manai M, Ben Fayala MN, et al (2013) Occurrence of plasmid-mediated quinolone resistance determinants and rmtB gene in Salmonella entericaserovarenteritidis and Typhimurium isolated from food-animal products in Tunisia. Foodborne Pathog Dis 10(9): 813-819.

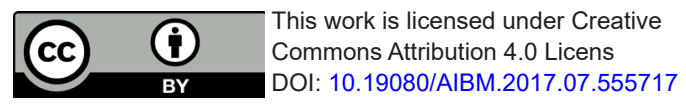

5. Chen S, Cui S, McDermott PF, Zhao S, White DG, et al. (2007) Contribution of target gene mutations and efflux to decreased susceptibility of Salmonella entericaserovar typhimurium to fluoroquinolones and other antimicrobials. Antimicrob Agents Chemother 51(2): 535-542.

\section{Your next submission with Juniper Publishers will reach you the below assets}

- Quality Editorial service

- Swift Peer Review

- Reprints availability

- E-prints Service

- Manuscript Podcast for convenient understanding

- Global attainment for your research

- Manuscript accessibility in different formats ( Pdf, E-pub, Full Text, Audio)

- Unceasing customer service

Track the below URL for one-step submission https://juniperpublishers.com/online-submission.php 